\title{
Des enjeux
}

\section{de genre à la (re)féminisation de la gouvernance scolaire} au Québec : une dynamique d'égalisation,
de rattrapage ou de dévalorisation?

\author{
Anastasie Amboulé Abath ${ }^{a}$
}

RÉSUMÉ. Cet article s'appuie sur des statistiques ministérielles pour examiner la question de la féminisation de la gestion scolaire au Québec et met en évidence dans quelles conditions celle-ci s'est effectuée. De façon spécifique, à travers une recherche documentaire, nous avons recueilli, généré et analysé des données administratives et prévisionnelles sur le personnel cadre des commissions scolaires (devenues des centres de services scolaires en 2020) et des écoles selon le sexe au Québec, de la fin des années 1950 à 2019, pour mieux saisir les enjeux de genre qui y sont liés. Dans cette perspective, une analyse sociohistorique retraçant les enjeux sexospécifiques de l'évolution des effectifs femmes/hommes occupant les postes de cadres scolaires a été réalisée. Notre démarche prend comme angle de lecture les étapes charnières de cette évolution, lesquelles semblent liées à des moments clés issus des grandes réformes éducatives. Les statistiques ministérielles guident nos analyses et permettent de repérer quand et comment les courbes statistiques se sont inversées et pourquoi.

ABSTRACT. This article uses ministerial statistics to examine the question of the feminization of school management in Quebec and highlights the conditions under which this was done. Specifically, through documentary research, we collected, generated and analyzed by sex administrative and forecast data on the management staff of school boards (which became school service centers in 2020) and schools in Quebec, from the late 1950s to 2019, to better understand the related gender issues. In this perspective, a sociohistorical analysis, that traces the gender issues of the evolution of the number of women and men occupying school management positions, was carried out. Our approach takes as a reading angle the pivotal stages of this development, that seem to be linked to key moments resulting from the major educational reforms. Ministerial statistics guide our analyses and enable to identify when and how the statistical curves have reversed, and why.

\section{Introduction}

La question de la féminisation des professions ou des tâches renvoie à de nombreux enjeux théoriques et sociaux. Les femmes sont de plus en plus présentes dans les postes de direction d'école et de gérance des commissions scolaires (devenues des centres de services scolaires en 2020) au Québec. Les statistiques récentes du ministère de l'Éducation et de l'Enseignement supérieur $(\mathrm{MEES})^{1}$ pour l'année 2014-2015 montrent que les femmes sont encore plus nombreuses et forment $67,18 \%$ des effectifs parmi les cadres d'école et de commission scolaire ${ }^{2}$ francophones, comparativement aux hommes, qui constituent 32,82\% (PERCOS-Bloc 2; MELS, 2015). L'objet de cet article théorique est de proposer une analyse sociohistorique qui retrace les enjeux sexospécifiques de l'évolution de la place des femmes et des hommes en gestion de l'éducation au Québec.

Pour y parvenir, et au-delà de la rareté des sources archivistiques, nous nous sommes inspirée des travaux de Claudine Baudoux (Baudoux, 1987, 1988, 1989, 1991, 1994, 2005; Baudoux et De la Durantaye, 1988; Baudoux et Girard, 1990; Baudoux

a Professeure, département des sciences de l'éducation, Université du Québec à Chicoutimi 
et Zaidman, 1992), des statistiques ministérielles ainsi que des autres sources et bases de données nous permettant de découvrir des données intéressantes sur la division genrée des postes de cadres scolaires au Québec.

Nous dresserons l'historiographie de la féminisation de la gestion scolaire au Québec depuis la fin des années 1950 à nos jours en accordant une attention particulière aux réversibilités sexuelles dans les fonctions de direction d'école et de gérance des commissions scolaires. Ensuite, nous tracerons les périodisations de basculement des effectifs dans les postes de cadres scolaires en matière d'obstacles et d'opportunités pour les femmes. Enfin, nous définirons le concept de féminisation dans les sciences sociales, puis discuterons de ses enjeux en gestion scolaire.

Il s'agit pour nous d'analyser comment les phénomènes de féminisation et de masculinisation des postes de cadres scolaires ont évolué et se sont mués au fil du temps. Les statistiques ministérielles nous guident dans la lecture de l'écart entre les sexes, nous permettent de repérer quand et comment les courbes statistiques se sont inversées, et pourquoi. Puis, les éléments sociologiques d'une féminisation nous permettront d'explorer les principaux enjeux qui accompagnent les tendances statistiques, les causes, les conséquences et les implications.

\section{Les retournements statistiques selon le genre en gestion scolaire}

Dans cette section, nous présentons une généalogie de l'évolution des données sur la répartition des hommes et des femmes en gestion de l'éducation en trois périodes: 1) avant les années 1960, 2) de la réforme des années 1960-2000 et 3) des années 2000 à nos jours.

D'abord, la répartition inégalitaire des pourcentages femmes/hommes dans les postes de direction d'établissements scolaires au Québec a connu un premier revirement lors de la modernisation du système scolaire dans les années 1960. Ce retournement statistique est perceptible dans le tableau 1, dont les données sont tirées d'enquêtes principalement compilées dans les travaux de Baudoux $(1994,1991,1989)$ et de Baudoux et Girard (1990).

Puis, au début des années 1990, les statistiques prévisionnelles du ministère de l'Éducation (MEQ) réalisées par Ouellette (2000) montrent une autre inversion de la tendance. Les statistiques obtenues en vertu de la Loi sur l'accès à l'information au ministère de l'Éducation et de l'Enseignement supérieur (Demande d'accès aux documents adressée au MEES, dossier 16310/16-88) en juillet 2016 pour l'année 2014-2015 permettent de brosser un portrait récent de la situation. Elles sont présentées au tableau 4.

\section{De la masculinisation à \\ la féminisation de la gestion scolaire au Québec}

Rappelons qu'au XXe siècle, l'enseignement est défini au Québec comme une profession féminine, mais le personnel enseignant n'a pas toujours été composé en majorité de femmes. Jusqu'à l'arrivée massive des membres des congrégations religieuses françaises au $\mathrm{XIX}^{\mathrm{e}}$ siècle, l'enseignement était surtout une affaire d'hommes (Dumont-Johnson et Fahmy-Eid, 1983, citées par Baudoux, 1994). Concernant la fonction de direction d'établissement scolaire au Québec, elle est marquée par deux figures historiques: l'ancêtre confessionnel (soit le supérieur d'une congrégation religieuse) et l'ancêtre laïque (soit le principal d'école) (Brassard, 2009).

Le retournement statistique des années 1960 vers la masculinisation du personnel de direction d'école est une étape marquante dans les mécanismes qui expliquent les enjeux de genre en gestion scolaire (Baudoux, 1994). 


\begin{tabular}{|c|c|c|c|c|}
\hline Années & Hommes & Femmes & Total & $\begin{array}{c}\% \text { des } \\
\text { femmes }\end{array}$ \\
\hline 1958-1959 & 1278 & 1833 & 3111 & 59 \\
\hline 1959-1960 & 1424 & 1974 & 3398 & 58 \\
\hline 1960-1961 & 1443 & 2008 & 3451 & 58 \\
\hline 1961-1962 & 1695 & 2152 & 3847 & 56 \\
\hline 1962-1963 & 1936 & 2318 & 4254 & 54 \\
\hline 1963-1964 & 2080 & 2309 & 4389 & 53 \\
\hline $1964-1965$ & 2561 & 2388 & 4949 & 48 \\
\hline 1965-1966 & 2197 & 1980 & 4177 & 47 \\
\hline 1966-1967 & 2873 & 2142 & 5015 & 43 \\
\hline 1967-1968 & 3193 & 2277 & 5470 & 42 \\
\hline 1968-1969 & 3398 & 2175 & 5573 & 39 \\
\hline 1969-1970 & 5195 & 3020 & 8215 & 37 \\
\hline 1970-1971 & 5374 & 2764 & 8138 & 34 \\
\hline 1971-1972 & 5798 & 2641 & 8439 & 31 \\
\hline $1972-1973$ & $\mathrm{NC}$ & $\mathrm{NC}$ & 4264 & $\mathrm{NC}$ \\
\hline 1973-1974 & $\mathrm{NC}$ & $\mathrm{NC}$ & 4435 & $\mathrm{NC}$ \\
\hline 1974-1975 & $\mathrm{NC}$ & $\mathrm{NC}$ & 4534 & $\mathrm{NC}$ \\
\hline $1975-1976$ & 3191 & 1481 & 4582 & 32 \\
\hline 1976-1977 & 2958 & 1289 & 4247 & 30 \\
\hline $1977-1978$ & 2946 & 1209 & 4155 & 29 \\
\hline 1978-1979 & 2924 & 1128 & 4052 & 28 \\
\hline 1979-1980 & 2900 & 1088 & 3988 & 27 \\
\hline 1980-1981 & 2925 & 1067 & 3992 & 27 \\
\hline 1981-1982 & 2834 & 1030 & 3864 & 27 \\
\hline 1982-1983 & 2802 & 985 & 3787 & 26 \\
\hline 1983-1984 & 2784 & 929 & 3713 & 25 \\
\hline 1984-1985 & 2678 & 852 & 3530 & 25 \\
\hline
\end{tabular}

Tableau 1 - Évolution de la répartition du personnel de direction selon le sexe dans les secteurs public et privé, catholique et protestant, au primaire et au secondaire, de 1958 à 1985 Source : Baudoux (1994, p. 24)

Un examen statistique des données du tableau 1 montre que le personnel de direction est d'abord en légère majorité féminine de 1958 à 1964. C'est en 1964-1965 qu'a eu lieu la première réversion. Comme le souligne Baudoux (1994), la décennie 1960 fut le temps fort de la masculinisation des postes de gestion scolaire. Ainsi, de 1964 à 1985, on remarque un mouvement de diminution progressive de la présence féminine à la tête d'établissements scolaires. Cette diminution des cadres féminines reste constante au cours des trois décennies suivantes puisque les femmes sont d'abord et avant tout considérées comme mères et épouses responsables de l'intendance familiale (Baudoux, 1988, 1989, 1991, 1994, 2005; Baudoux et De la Durantaye 1988; Baudoux et Girard, 1990).

En effet, la déconstruction statistique de la catégorie personnelle de direction à travers la lente disparition des directrices est d'ailleurs qualifiée de "péril féminin en éducation» par Baudoux (1994). La masculinisation des cadres d'établissement est soutenue par les politiques, qui légitimaient les actions des hommes et qui prônaient des discours sur la nature des femmes en tentant de réglementer leur accès aux postes de direction.

L'éviction des directrices survient à un moment clé de l'histoire du système scolaire du Québec. En 1961, le gouvernement a institué la Commission royale d'enquête sur l'enseignement dans la province de Québec, aussi appelée commission Parent, qui a publié les différents tomes de son rapport entre 1963 et 1966 (Commission Parent, 1964). Cette période coïncide avec la création d'un ministère de l'Éducation au Québec, en mai 1964. Le début des années 1960 est donc une période où la société québécoise est en pleine ébullition émancipatrice, caractérisée par un changement de valeurs et de mentalités. Les forces progressistes ont engagé la société québécoise dans 
une voie accélérée de modernisation. C'est la Révolution tranquille; l'ère de la réforme en profondeur du système scolaire actuel.

Il est difficile d'expliquer la diminution des femmes parmi les membres du personnel de direction à cette période où la société québécoise prône le principe de l'égalité entre les sexes. L'historiographie des données compilées par Baudoux (1994) depuis les années 1950 jusqu'au début des années 1990 au Québec a montré une masculinisation de la gestion scolaire au moment de la modernisation du système scolaire. Selon Baudoux (1989, 1991), deux phénomènes sociaux auraient accéléré la masculinisation des postes de gestion scolaire au Québec lors de la Révolution tranquille : la laïcisation de l'éducation, qui a évacué des écoles les religieux - mais encore plus les religieuses (voir les données du tableau 2), et la mixité scolaire, qui a eu pour conséquence la préférence accordée aux hommes plutôt qu'aux femmes pour diriger les nouvelles écoles mixtes.

Toujours selon Baudoux, jusqu'à l'introduction des programmes d'accès à l'égalité, la bureaucratisation qui a suivi la création du ministère de l'Éducation du Québec en 1964 a entrainé une survalorisation dans le rôle de direction d'école des tâches administratives plutôt que pédagogiques; a instauré une division sexuelle du travail en accordant les postes de direction d'école aux candidats masculins et les postes de direction adjointe à certaines candidatures féminines; et a éloigné les femmes de l'admissibilité aux postes de direction par le biais d'exigences plus élevées de formation (Baudoux, 1987, 1988, 1990). La minorité de femmes qui étaient nommées directrices travaillaient au primaire, où elles assuraient une image prégnante de la mère (Baudoux, 1988, 1994).

Cette auteure a également constaté que le style de gestion alors préconisé dans le monde de l'éducation au Québec était de type masculin et que la bureaucratisation des structures éducatives qui a précédé le rapport Parent, à la fin des années 1960 et au début des années 1970, a favorisé la masculinisation des effectifs. Baudoux (1994) qualifie cette période comme celle de la «neutralisation des femmes travaillant dans le secteur de l'éducation» puisqu'elle se caractérise par de nouvelles pratiques qui «invisibilisent les femmes» et favorisent le «recours au générique masculin» comme équivalent du neutre (p.118). Elle estime qu'avec la mixité, tous les progrès antérieurement réalisés par les femmes et la place occupée par les religieuses ont été perdus, dans la mesure où la direction d'une école mixte revenait de préférence aux hommes. Autrement dit, la laïcisation du système scolaire, la généralisation de la mixité et la bureaucratisation de la gestion scolaire seraient des facteurs associés à la masculinisation du personnel de direction d'école (Baudoux, 1989).

Cet état de fait appuie l'hypothèse d'une discrimination systémique qui se perpétue, même si les arguments évoqués pour justifier la sous-représentation des femmes étaient que «les enseignantes ne veulent pas des postes de direction; les responsabilités familiales nuisent à la carrière; les postes sont octroyés selon la compétence; la discrimination, c'est chose du passé» (Baudoux, 1994, p. 9).

La faible représentation féminine est surtout évoquée à travers les commentaires sur la moindre ambition des femmes, sur le manque de confiance ou sur l'absence de compétences, ce qui renvoie le problème aux femmes elles-mêmes et qui nie complètement toute composante extérieure liée à l'enjeu des femmes dans la société et dans les organisations (famille, conventions sociales). Les femmes étant plus nombreuses en enseignement, leur absence aux fonctions dirigeantes passait pour normale. Les principes affichés sont marqués par des normes genrées inégalitaires qui reproduisent dans leurs actes et dans leurs pensées des pratiques discriminantes en faveur des hommes. Le discours à la mode était que la finalité du travail féminin se limitait aux actions domestiques et à l'intendance familiale.

Dans ce contexte, la perception d'un salaire d'appoint et la perspective de faire carrière n'étaient pas envisagées ni encouragées. Les qualités individuelles des femmes ne suffisaient pas, dans la plupart des cas, à les faire accéder à des postes décisionnels. Des mécanismes invisibles, subtils et informels ont handicapé de nombreuses carrières féminines dans le monde de l'éducation. À travers des pratiques sociales sexistes, les stéréotypes féminins étaient perçus comme contraires à l'accès à la promotion et à l'exercice de l'autorité. Les cooptations misogynes et les modèles très masculinisés de gestion soutenus par le cumul de préjugés sexistes, 
culturels, comportementaux et surtout organisationnels qui sévissaient en milieu professionnel ont été de véritables embûches pour les enseignantes aspirant à des postes de direction (Baudoux, 1994, 2005; Baudoux et De la Durantaye, 1988; Baudoux et Zaidman, 1992). L'image du gestionnaire efficace ressemble à celle d'un homme affirmé, fort et compétitif. Ces caractéristiques font que les femmes ont eu de la difficulté à se reconnaître comme des gestionnaires légitimes.

Faisant face à la réalité d'un univers professionnel souvent hostile, certaines enseignantes, résignées, ont abandonné leur projet professionnel pour se consacrer à leur vie familiale et sociale. D'autres, décidées à se faire reconnaître, ont fait carrière en surmontant nombre de difficultés professionnelles.

Une discordance apparait nettement entre le nombre de femmes en enseignement, d'une part, et leur effectif dans les postes de direction, de l'autre. La période de 1965 à 1989 est pourtant reconnue comme celle où l'on a observé une forte diversification des mobilisations féministes pour l'égalité3.

Données prévisionnelles du MEQ

pour les années 1989 à 1998

Les données présentées dans le tableau 2 indiquent que les effectifs de direction d'école et de gérance des commissions scolaires sont à la baisse depuis 19891998; ils sont passés de 3756 personnes en début de période à 3274 personnes neuf années plus tard. Durant cette période, le personnel de direction compte de plus en plus de femmes; de $28 \%$ en 19891990, on passe à $41 \%$ au 30 septembre 1998. Ces données montrent une ségrégation occupationnelle puisque la surreprésentation de femmes en enseignement ne suffit pas pour leur garantir une équitable représentation dans les postes de pouvoir et pour transcender la discrimination systémique qui limite leur accès (voir section 1.2). À bien des égards, le portrait statistique du personnel de direction d'école établit la situation de sexisme ambiant.

\begin{tabular}{|c|c|c|c|c|c|}
\hline Années & Total & $\begin{array}{c}\text { Effectif } \\
\text { hommes }\end{array}$ & $\begin{array}{c}\text { Effectif } \\
\text { femmes }\end{array}$ & \% hommes & \% femmes \\
\hline $1989-1990$ & 3756 & 2709 & 1047 & 72,1 & 27,9 \\
\hline $1990-1991$ & 3785 & 2690 & 1095 & 71,1 & 28,9 \\
\hline $1991-1992$ & 3762 & 2634 & 1128 & 70,0 & 30,0 \\
\hline $1992-1993$ & 3713 & 2549 & 1164 & 68,7 & 31,3 \\
\hline $1993-1994$ & 3664 & 2477 & 1187 & 67,6 & 32,4 \\
\hline $1994-1995$ & 3596 & 2398 & 1198 & 66,7 & 33,3 \\
\hline $1995-1996$ & 3554 & 2308 & 1246 & 64,9 & 35,1 \\
\hline $1996-1997$ & 3479 & 2215 & 1264 & 63,7 & 36,3 \\
\hline $1997-1998$ & 3385 & 2075 & 1310 & 61,3 & 38,7 \\
\hline $1998-1999$ & 3274 & 1933 & 1341 & 59,0 & 41,0 \\
\hline
\end{tabular}

Tableau 2 - Personnel de direction d'école et de gérance des commissions scolaires prévu de 1989 à 1998 Source : Ouellette (2000, tableau 1)

Autres sources et bases de données utilisées pour éclairer l'analyse: données prévisionnelles du MEQ pour les années 2000

Le tableau 3 résume les projections du personnel de direction et de gérance des commissions scolaires de 1999 à 2010. Ces projections, calculées en fonction des vagues de départs à la retraite et de la diminution du nombre d'élèves des commissions scolaires, montrent une réversion possible en 2004-
2005 et qu'il devrait y avoir plus de femmes que d'hommes aux postes de direction ou de direction adjointe des écoles.

Puis, vers 2010-2011, les femmes seraient majoritaires dans une proportion avoisinant $54 \%$. On observe, à partir de cette conjoncture, que la féminisation de la gestion scolaire au Québec est un processus contextuel, situationnel et conjoncturel qui révèle de nombreux enjeux et logiques sociaux complexes. 


\begin{tabular}{|c|c|c|c|c|c|}
\hline Années & Total & $\begin{array}{c}\text { Effectif } \\
\text { hommes }\end{array}$ & $\begin{array}{c}\text { Effectif } \\
\text { femmes }\end{array}$ & \% hommes & \% femmes \\
\hline $1999-2000$ & 3196 & 1841 & 1355 & 57,6 & 42,4 \\
\hline $2000-2001$ & 3214 & 1796 & 1418 & 55,9 & 44,1 \\
\hline $2001-2001$ & 3208 & 1737 & 1471 & 54,1 & 45,9 \\
\hline $2002-2003$ & 3190 & 1673 & 1517 & 52,4 & 47,6 \\
\hline $2003-2004$ & 3159 & 1610 & 1549 & 51,0 & 49,0 \\
\hline $\mathbf{2 0 0 4 - 2 0 0 5}$ & $\mathbf{3 1 2 7}$ & $\mathbf{1 5 5 5}$ & $\mathbf{1 5 7 2}$ & $\mathbf{4 9 , 7}$ & $\mathbf{5 0 , 3}$ \\
\hline $2005-2006$ & 3099 & 1512 & 1587 & 48,8 & 51,2 \\
\hline $2006-2007$ & 3067 & 1474 & 1593 & 48,1 & 51,9 \\
\hline $2007-2008$ & 3021 & 1433 & 1588 & 47,4 & 52,6 \\
\hline $2008-2009$ & 2968 & 1394 & 1574 & 47,0 & 53,0 \\
\hline $2009-2010$ & 2909 & 1354 & 1555 & 46,5 & 53,5 \\
\hline $\mathbf{2 0 1 0 - 2 0 1 1}$ & $\mathbf{2 8 5 2}$ & $\mathbf{1 3 1 8}$ & $\mathbf{1 5 3 4}$ & $\mathbf{4 6 , 2}$ & $\mathbf{5 3 , 8}$ \\
\hline
\end{tabular}

Tableau 3 - Personnel de direction d'école et de gérance des commissions scolaires prévu de 1999 à 2011 Source : Ouellette (2000, tableau 3)

Après la réforme des années 1960, d'autres changements ont eu lieu en matière de gestion en éducation, par exemple la tenue d'États généraux en 1995, dont le rapport (Commission des États généraux sur l'éducation, 1996) est à l'origine de l'intérêt porté au rôle que jouent les directions d'établissement annoncé à l'automne 1997 par la sanction de la Loi modifiant la Loi sur l'instruction publique et diverses dispositions législatives (Québec, MEQ 1997), qui consacrait l'autonomie des établissements scolaires en énonçant les grands principes de la politique de décentralisation du système éducatif et la complexification de la fonction de direction d'établissement.

Cette loi a engendré la professionnalisation de la fonction de direction d'un établissement d'enseignement, des mécanismes pour la sélection des directions d'école de même qu'une préoccupation accrue pour leur qualification (MELS, 2008). C'est ainsi qu'en 2001, le Règlement sur les conditions d'emploi des gestionnaires des commissions scolaires (Québec, 2001) fixait l'obligation d'une formation de deuxième cycle en gestion scolaire et marquait, par le fait même, l'importance de la formation continue pour le personnel de direction.

Dans l'ensemble, cet aperçu sociohistorique nous a permis de prendre acte de l'évolution de la situation des femmes depuis la fin des années 1950 à ce jour, notamment de constater que d'énormes progrès ont été accomplis au cours des dernières années et que les femmes, de plus en plus diplômées au diplôme d'études supérieures spécialisées (DESS) en administration scolaire, sont entrées de plainpied dans des postes de gestion scolaire, sphère auparavant occupée par les hommes, et ce, même si l'histoire de leur accès est loin d'être terminée.

\section{Un portrait évolutif de \\ la féminisation de la gestion scolaire}

Les données statistiques compilées sur la présence des femmes dans la gestion scolaire en 2014-2015 confirment la féminisation effective des postes de cadres scolaires. Parmi les 3346 gestionnaires scolaires des commissions scolaires francophones, on dénombre 2248 femmes (67,18\%), contre 1098 hommes $(32,82 \%)$ occupant des postes de gestion.

Il n'est pas de notre propos de nous prononcer sur les différents niveaux de participation à la gestion scolaire des femmes. Notre analyse cherche plutôt à étayer l'hypothèse selon laquelle la surreprésentation des femmes dans ces fonctions observées aujourd'hui résulte d'un long processus de construction sociale et trouve son explication dans les changements sociaux survenus dans la société québécoise au cours des dernières décennies. 


\begin{tabular}{|ccccc|}
\hline Années & $\begin{array}{c}\text { Effectif } \\
\text { hommes }\end{array}$ & $\begin{array}{c}\text { Effectif } \\
\text { femmes }\end{array}$ & Total & $\begin{array}{c}\text { \% des } \\
\text { femmes }\end{array}$ \\
\hline $\mathbf{1 9 5 8 - 1 9 5 9}$ & $\mathbf{1 2 7 8}$ & $\mathbf{1 8 3 3}$ & $\mathbf{3 1 1 1}$ & $\mathbf{5 9}$ \\
$\mathbf{1 9 6 5 - 1 9 6 6}$ & $\mathbf{2 1 9 7}$ & $\mathbf{1 9 8 0}$ & $\mathbf{4 1 7 7}$ & $\mathbf{4 7}$ \\
$\mathbf{1 9 6 9 - 1 9 7 0}$ & 5195 & 3020 & 8215 & 37 \\
$\mathbf{1 9 7 5 - 1 9 7 6}$ & 3191 & 1481 & 4582 & 32 \\
$\mathbf{1 9 7 9 - 1 9 8 0}$ & 2900 & 1088 & 3988 & 27 \\
$\mathbf{1 9 8 4 - 1 9 8 5}$ & 2678 & 852 & 3530 & 25 \\
$\mathbf{1 9 8 9 - 1 9 9 0}$ & 2690 & 1095 & 3785 & 29 \\
$\mathbf{1 9 9 4 - 1 9 9 5}$ & 2308 & 1246 & 3554 & 35 \\
$\mathbf{1 9 9 9 - 2 0 0 0}$ & 1796 & 1418 & 3214 & 44 \\
$\mathbf{2 0 0 4 - 2 0 0 5}$ & $\mathbf{1 5 5 5}$ & $\mathbf{1 5 7 2}$ & $\mathbf{3 1 2 7}$ & $\mathbf{5 0 , 3}$ \\
$\mathbf{2 0 0 9 - 2 0 1 0}$ & $\mathbf{1 3 1 8}$ & 1534 & 2852 & 53,8 \\
$\mathbf{2 0 1 4 - 2 0 1 5}$ & $\mathbf{1 0 9 8}$ & $\mathbf{2 2 4 8}$ & $\mathbf{3 3 4 6}$ & $\mathbf{6 7 , 1 8}$ \\
\hline
\end{tabular}

Tableau 4-Évolution du personnel cadre selon le sexe de 1958 à 2015 (récapitulatif)

Aussi intéressantes soient-elles, ces données sont dénuées d'intérêt sociologique si on ne peut y juxtaposer une composante explicative. Quelques travaux se sont intéressés aux barrières limitant l'accès des femmes aux postes de directrice, voire de directrice adjointe dans les écoles secondaires (Baudoux, 1987, 1994; Baudoux et Girard, 1990; Cacouault et Combaz, 2007; Cacouault-Bitaud, 1999; De la Durantaye, 1989; Héon et Kamyap, 2018; Héon et Mayrand, 2003). Il ressort de ces études de nombreux facteurs discriminatoires qui ont eu des impacts sur le processus d'accès et de promotion des femmes. Le tableau récapitulatif ci-dessus permet certes de dresser un bilan positif de la représentation actuelle des femmes, mais ne nous permet pas de comprendre quelles embûches ont disparu et quelles sont celles qui sont toujours présentes. Un des faits importants reste aussi celui de savoir s'il s'agit de simples retournements statistiques ou d'une évolution prévisible.

Héon et Kamyap (2018) ont établi des liens étroits entre les pratiques de gestion des ressources humaines dans le secteur d'activité de l'administration scolaire et le contexte sociopolitique dans lequel a évolué la représentation des femmes dans ce domaine. Selon ces auteures, trois périodes principales permettent de comprendre l'évolution de la place des femmes en administration scolaire dans les commissions scolaires de 1954 à 2016 :

- 1954 à 1990 : Exclusion - coincer sous le plafond de verre;

- 1990 à 2000 : Progression - briser le plafond de verre;

- 2000 à aujourd'hui : Croissance - être avec les autres au dehors du plafond de verre.
Elles estiment qu'il a fallu attendre 60 ans pour atteindre la zone paritaire de 40/60. Selon le Consiel du statut de la femme, cette « zone paritaire ou zone de mixité égalitaire dans un secteur d'activité : le nombre de femmes ou d'hommes ne descend jamais sous le taux de $40 \%$ et ne dépasse jamais $60 \%$ » (Conseil du statut de la femme $(2015$, p. 8). À titre d'exemple, dans le domaine politique, «la mixité égalitaire existe lorsque les personnes élues de chaque sexe occupent de $40 \%$ à $60 \%$ des sièges » (CSF, 2015, p. 7). Alors, comment expliquer l'inversement des genres au début du XXIe siècle? Quels sont les facteurs favorables à la féminisation des postes de cadres scolaires au Québec? Mais tout d'abord: qu'entend-on par féminisation?

\section{Le concept de féminisation dans les sciences sociales}

\section{La féminisation}

De façon générale, les recherches contemporaines en sciences humaines et sociales sur les réversibilités sexuelles s'inscrivent le plus souvent dans le sens d'une féminisation. Le processus de la féminisation des professions a suscité un débat scientifique où se sont confrontés différents points de vue interprétatifs en sociologie du travail et en sociologie des professions.

Ce processus s'inscrit dans un contexte sociétal structuré par une combinaison de rapports spécifiques entre les sexes. Il met en exergue la reconfiguration des sexes et rend compte de la confrontation des différents rapports de pouvoir et des exigences professionnelles. La féminisation désigne plus étroitement «l'inversion du genre (Guichard-Claudic et collab., 
2008). Ainsi, elle traduit l'effritement de bastions masculins par l'arrivée des femmes et, dans un sens beaucoup plus restreint, elle désigne l'ensemble des mécanismes institutionnels qui aboutissent à l'évolution des carrières de femmes et qui permettent leur accès aux postes à responsabilités plus complexes.

La féminisation désigne aussi les multiples embûches rencontrées par des femmes lorsqu'elles choisissent d'entrer dans des univers dont le prestige se conjugue au masculin (Lapeyre, 2005, 2006). Zaidman (1986) distingue trois usages principaux du concept de féminisation :

1) La féminisation peut d'abord être comprise comme un élément d'une «dynamique d'égalisation» qui efface ou atténue un retard historique dommageable;

2) Elle peut ensuite renvoyer à une inversion quantitative, lorsqu'« une profession tout entière est investie en force par les femmes et désertée par les hommes » (p. 281). Dans cette optique, il n'est pas rare qu'une corrélation directe soit établie entre la féminisation de la profession concernée et sa dévalorisation sociale;

3) La féminisation peut recomposer la frontière entre métiers féminins et masculins en subvertissant la hiérarchie des sexes. Elle renvoie alors à l'ouverture discrète vers des femmes qui, progressivement, contribuent à transformer les représentations et les comportements masculins.

Selon ces logiques sous-jacentes, la notion de féminisation des groupes professionnels ne correspond pas forcément aux mêmes réalités. D'autres perspectives se centrent sur les phénomènes de recomposition des rapports sociaux de sexe dans la sphère professionnelle (Baudoux, 1994; Cacouault-Bitaud, 2001; Lapeyre, 2005; Malochet, 2005, 2006).

Enfin, la féminisation d'une profession peut également signifier l'accession des femmes dans des bastions masculins synonymes de prestige et de pouvoir (Le Feuvre, 1998, cité dans Lapeyre, 2005).

\section{La masculinisation}

En sens inverse, la masculinisation permet de cerner un mouvement ascendant dans les taux de masculinité des emplois pouvant se traduire de trois manières :

1) un secteur largement masculin qui devient de plus en plus masculin et qui s'accompagne soit de l'augmentation des ghettos d'emplois, sinon du refoulement des femmes dans la sphère domestique;

2) une entrée progressive des hommes dans un ghetto d'emplois féminins;

3) une entrée massive des hommes dans un secteur traditionnellement féminin.

Toutefois, le terme masculinisation pourrait également traduire une masculinisation des pratiques, des valeurs et des comportements qui correspondraient à une masculinisation statistique. Dans ce cas, la masculinisation signifierait une transformation des caractéristiques autrefois féminines d'une profession et une valorisation sociale des caractéristiques masculines (Baudoux, 1994).

La question de la féminisation des métiers ou des tâches renvoie donc à un certain nombre d'enjeux théoriques et sociaux qui contribuent à la réflexion sur les rapports sociaux de sexe dans des contextes organisationnels en mutation. Cela implique parfois pour les postes décisionnels une certaine «hostilité à l'arrivée des femmes, étant donné que les stratégies masculines visent à recréer l'ordre de genre, soit en confirmant leur virilité, soit en faisant fonctionner de façon récurrente le couple féminisation/dévalorisation » (Guichard-Claudic et collab., 2008, p. 17).

Dans cet ordre d'idées, la féminisation est constituée en problème et les femmes sont tenues pour responsables des dysfonctionnements de la profession ou, tout au moins, de la détérioration de son image. Toutefois, la féminisation peut également être vue comme le résultat de la désaffection des hommes à l'endroit d'un métier, associé à une dégradation des conditions d'exercice de la profession (Héon et collab., 2008). 
Ainsi, au-delà du phénomène purement statistique, l'expression féminisation recouvre une réalité plus complexe; elle doit être comprise comme le processus par lequel un secteur à dominante masculine tend à devenir féminin; processus, en somme, par lequel tend à se reproduire la division sociale des sexes.

En comprenant mieux le comment, le pourquoi, les origines et les modalités de la féminisation de certains secteurs, il semble qu'on soit plus à même de saisir les dynamiques qui sous-tendent la reproduction des clivages entre les hommes et les femmes (Héon et collab, 2008). Dès lors, le simple fait que les femmes accèdent à un fief masculin n'est pas suffisant pour parler de féminisation. Les faits historiques ont montré qu'il existe parfois des décalages temporels importants entre le moment où les femmes accèdent à un secteur et celui où elles y seront suffisamment nombreuses pour ne plus être invisibles.

Dans le cas actuel des travaux en administration de l'éducation, les femmes sont invisibles. En effet, la surreprésentation féminine dans l'effectif des cadres scolaires reste un phénomène marginal, (les données des recherches récentes n'indiquent pas les pourcentages sexo-spécifiques) alors qu'il s'agit d'un phénomène collectif qui devrait avoir une résonance sociale particulière pour qu'on parle de féminisation.

\section{L'acceptation quantitative et les enjeux de la féminisation de la gestion scolaire}

La féminisation des cadres scolaires est un phénomène statistique démontré par une augmentation significative de la proportion de femmes dans les positions de gestionnaires scolaires. Elle désigne par le fait même l'augmentation marquée du nombre d'écoles dirigées par les femmes dans les fonctions de direction et de direction adjointe d'école partout au Québec depuis 2004.

À la même période, une étude réalisée en 2005-2006 par la Fédération québécoise des directions d'établissement d'enseignement (Fortin, 2006), intitulée L'insertion professionnelle des nowvelles directions d'établissement d'enseignement, concluait que de moins en moins de candidats se présentaient aux postes annoncés et que plus des deux tiers du personnel de direction en poste depuis moins de cinq ans avaient au moins une fois remis en question leur décision d'occuper un tel poste.
Les motifs les plus invoqués pour justifier cette remise en question étaient la lourdeur de la tâche, la quantité de paperasse à remplir et la complexification des problèmes à résoudre (gestion des ressources humaines, financières et matérielles; diversité des clientèles et des élèves en difficulté; relations parfois difficiles avec les parents; problèmes de violence et de toxicomanie; etc.).

Plus récemment, d'autres recherches ont clairement démontré des écarts importants entre le temps de travail réel et le temps de travail souhaité des gestionnaires scolaires, ainsi que la complexification de la tâche, qui s'alourdit avec de nouveaux défis et responsabilités (Bernatchez, 2011; Poirel et Yvon, 2009; St-Germain, 2013 2011; MELS, 2006). Ces aspects socio-organisationnels liés à l'exercice de la fonction de direction d'établissement soutiendraient-ils le constat selon lequel «lorsqu'une profession ou un métier se féminise, c'est que les conditions de travail s'y sont sérieusement détériorées ou que le statut social qui leur est associé a fortement diminué, alors que, lorsqu'ils se masculinisent, c'est le contraire qui arrive» (Héon et collab., 2008)?

Nous avons constaté, dans notre parcours de sens, que les usages contemporains du vocable féminisation dans son saisissement scientifique prêtent à confusion. Une série de constats épars et non exhaustifs dans la littérature consultée reconnait en tout temps le caractère dynamique des rapports sociaux de sexe. C'est ainsi que cette notion devient le porte-étendard des diverses acceptions, selon les usages établis par Zaidman (1986). Ce terme, qui désigne d'abord un constat statistique puisqu'on parle plus volontiers du taux de féminisation que du taux de masculinisation, peut ainsi traduire deux usages :

1) une féminisation entendue comme dynamique d'égalisation et de rattrapage présumé d'un retard historique et donc une marche vers une égalité de droits et une égalité de nombre; et

2) une féminisation synonyme de transformation des caractéristiques d'une profession par l'arrivée des femmes et, donc, une forme de domination quantitative dans un secteur déserté par les hommes.

Ces dimensions non tranchées portent sur des points permettant de comprendre les évolutions affectant sans cesse les rapports sociaux de sexe dans le milieu 
professionnel. Cependant, cette notion est indéniablement source de richesses, notamment parce qu'elle renvoie à une multitude de facettes qui se complètent pour participer à la construction d'un terme évolutif susceptible de rendre compte des reconversions statistiques survenues dans diverses professions visant l'abolition de tout parti pris dans l'acception du terme féminisation comme dynamique d'égalisation, de rattrapage ou de dévalorisation.

C'est ainsi qu'au cours des dernières décennies, les domaines d'application de la notion de féminisation se sont multipliés et ont enrichi l'analyse des réversibilités sexuelles en sociologie du travail et des professions.

\section{Les éléments d'une féminisation à la fois statistique et sociologique}

Les derniers chiffres sur les effectifs femmes/ hommes ayant le statut professionnel supérieur dans les organismes scolaires en 2015 peuvent paraître importants à première vue, si on les compare à ceux d'autres secteurs d'activité au Québec. Cependant, il faut se rappeler que le système d'éducation est un secteur majoritairement et traditionnellement féminin. Si les femmes sont proportionnellement plus engagées à des postes de cadres scolaires, elles se retrouvent surtout et encore majoritairement au sein des directions d'écoles primaires et des directions adjointes d'écoles secondaires. Cette féminisation semble suggérer que les cadres féminines seraient tenues d'exercer, en plus des responsabilités formelles inhérentes au poste, une fonction supplémentaire, cachée, en partie contradictoire, reliée au genre féminin. Ce serait en tant que femmes, dotées d'une nature différente de celle des hommes, que les gestionnaires féminines auraient des élèves plus jeunes et plus d'élèves ayant un handicap, comme l'avait déjà constaté Baudoux dans les années 1980.

Si les hommes sont en moins grand nombre que les femmes à des postes cadres des écoles, le phénomène de faible représentation revêt un sens particulier, car ils sont plus nombreux dans les postes de commande des commissions scolaires, où se prennent les décisions importantes. La typologie de Kelleher (2011) illustre différents aspects de la féminisation de la gestion scolaire au Québec. Elle envisage deux types de féminisation: une statistique et l'autre sociologique.
Sur le plan statistique, la féminisation met en exergue le portait global du nombre de femmes cadres en milieu scolaire, et s'articule autour de données chiffrées mettant de l'avant le calcul des pourcentages d'hommes et de femmes dans les postes de cadres scolaires. Toutefois, ce bilan statistique n'explique pas tout. Quant au plan sociologique, elle fait référence à l'augmentation de la présence des femmes dans ces fonctions et examine cette féminisation en explorant les principaux enjeux qui accompagnent les tendances statistiques, les causes, les conséquences et les implications. Ces deux aspects sont éclairants dans la compréhension du processus de féminisation actuelle de la gestion scolaire au Québec.

\section{Conclusion}

Le présent article propose une réflexion sur le phénomène de la féminisation des cadres scolaires à partir de l'étude circonstanciée des statistiques ministérielles et des éléments d'interprétation sociohistorique. En administration de l'éducation, rares sont les travaux récents qui se sont intéressés au phénomène de la féminisation des cadres scolaires. Il est vrai que c'est un phénomène organisationnel récent, qui, pour nombre de chercheurs et chercheuses, est en marge des dynamiques contextuelles d'exercice qui sous-tendent ce statut professionnel légalement reconnu.

Devant un tel constat, la féminisation des cadres scolaires peut être perçue comme une sorte d'épiphénomène, peu utile finalement pour comprendre les enjeux sexospécifiques de ce statut socioprofessionnel.

Cette contribution visait à fournir un éclairage sur le concept de la féminisation de la gestion scolaire en prenant appui sur les usages de cette notion en sciences humaines et sociales ainsi que sur les différents retournements statistiques. La littérature montre sans équivoque l'importance de cette notion dans différents domaines d'activités professionnelles. La force du concept de féminisation repose, entre autres aspects, dans le fait qu'il est englobant et qu'il s'adapte à une variété de ségrégations genrées vécues par les femmes et les hommes dans l'activité professionnelle. Toutefois, cette force constitue également sa faiblesse puisque son transfert dans le contexte spécifique de la gestion scolaire au Québec demeure difficile à cerner. 
On peut sans doute imputer ce phénomène aux transformations sociales survenues dans la société québécoise au cours des dernières décennies ou à l'évolution des conditions d'exercice de la fonction de direction d'école. Les constats émis par divers auteures et auteurs ont mis en évidence la nécessité de redéfinir, voire d'élargir les contours de ce concept polysémique afin de faciliter son opérationnalisation des éléments d'interprétation différenciée selon le sexe des effectifs femmes/hommes en gestion scolaire au Québec.

Il nous est inapproprié, malgré les problématiques particulières connues du personnel de direction d'école et les transformations à la marge de pratiques professionnelles des gestionnaires scolaires, de voir une certaine « désertion » des hommes pour parler d'une dévalorisation de la fonction de direction d'école dans le domaine de l'enseignement, très féminisé.
Néanmoins, les dimensions sexospécifiques proposées dans la littérature permettent de s'approprier le concept après ce parcours de sens, d'en tirer le meilleur parti possible et, à terme, d'en faire une interprétation porteuse de sens traduisant cette réalité bien québécoise en gestion de l'éducation.

Nous sommes conscientes qu'il s'agit d'un aspect singulier de la recherche en administration scolaire et nous croyons qu'il était utile de suggérer les liens entre les définitions du mot féminisation, ses principales dimensions et ses usages actuels dans le champ de l'administration de l'éducation au Québec. Cet exercice nous a permis de cerner les contours de cette (re)féminisation de la gestion scolaire dans les commissions scolaires francophones du Québec.

\section{NOTES}

1 Selon l'année mentionnée, le ministère a porté le nom de ministère de l'Éducation du Québec (MEQ), ministère de l'Éducation, du Loisir et du Sport (MELS) ou ministère de l'Éducation et de l'Enseignement supérieur (MEES).

2 Dans le cadre de cet article, l'expression gestionnaire scolaire désigne à la fois les directrices et directeurs d'école et le personnel de gérance dans les commissions scolaires. Il s'agit de toute personne qui assure une fonction de direction, d'encadrement et de leadership dans la gouvernance opérationnelle et stratégique au service de la mission éducative d'une organisation d'enseignement.

3 https://histoiredesfemmes.quebec

\section{RÉFÉRENCES}

Baudoux, C. (1987). Caractéristiques de gestion des directrices d'écoles secondaires. Revne des sciences de l'éducation, 13(3), 385-406. https://doi.org/10.7202/900572ar

Baudoux, C. (1988). Sexualisation des tâches dans les postes de direction du primaire. Recherches féministes, 1(1) 61-77. https://doi.org/10.7202/057499ar

Baudoux, C. (dir.). (1989). Féminisation et masculinisation de la gestion. Cabiers de recherche du GREMF, 29.

Baudoux, C. (1991). Effets de la laïcisation et de la mixité sur le nombre de femmes responsables d'établissements scolaires du Québec de 1958 à 1985. Revue des sciences de l'éducation, 17(1), 1-24. https://doi.org/10.7202/900684ar

Baudoux, C. (1994). La gestion en éducation : une affaire d'hommes ou de femmes? Presses Inter Universitaires.

Baudoux, C. (2005). La passion de l'université : les femmes cadres dans la gestion collégiale et bureaucratique. Presses Inter Universitaires.

Baudoux, C. et De la Durantaye, C. (1988). La femme de l'organisation. PUQ.

Baudoux, C. et Girard, S. (1990). Sélection de candidatures féminines dans les postes de direction d'écoles et de collèges. Revue des sciences de l'éducation, 16(2), 163-183. https://doi.org/10.7202/900659ar

Baudoux, C. et Zaidman, C. (1992). Égalité entre les sexes : mixité et démocratie. L'Harmattan.

Bernatchez, J. (2011). La formation des directions d'établissement scolaire au Québec : apprendre à développer un savoir-agir complexe. Télescope, 17(3), 158-175. https://telescope.enap.ca/Telescope/docs/Index/Vol_17_no_3/Telv17n3_bernatchez.pdf 
Brassard, A. (2009). De principal d'école ou supérieur à directeur d'établissement au primaire et au secondaire (1840-2009). Le Point en administration de l'éducation, 12(1) 16-19.

Cacouault, M. et Combaz, G. (2007). Hommes et femmes dans les postes de direction des établissements secondaires : quels enjeux institutionnels et sociaux? Revue francaise de pédagogie, 158, 5-20. https://doi.org/10.4000/rfp.358

Cacouault-Bitaud, M. (1999). Égalité formelle et différenciation des carrières entre hommes et femmes chez les enseignants du second degré. Revue de l'TRES, 29(1), 95-129. http://www.ires.fr/index.php/publications-de-l-ires/item/2819-egalite-formelle-etdifferenciation-des-carrieres-entre-hommes-et-femmes-chez-les-enseignants-du-second-degre

Cacouault-Bitaud, M. (2001). La féminisation d'une profession est-elle le signe d'une baisse de prestige? Travail, genre et sociétés, 1(5), 93-116. https://doi.org/10.3917/tgs.005.0091

Commission des États généraux sur l'éducation. (1996). Rénover notre système d'éducation : dix chantiers prioritaires [rapport final]. Gouvernement du Québec. https://numerique.banq.qc.ca/patrimoine/details/52327/

39893?docref=jna4nV-2gsoRhomFXAskqw

Commission Parent. (1964). Rapport Parent (tome II). PUQ. https:// numerique.banq.qc.ca/patrimoine/ details/52327/4007670?docref=sTHIELOup1IeZ4CM6c_luQ

Conseil du statut de la femme (2015). Les femmes en politique: en route vers la parité. https:/ / www.csf.gouv.qc.ca/ wp-content/uploads/avis_femmes_et_politique_web2.pdf

De la Durantaye, C. (1989). L'alternance féminin/masculin en éducation au Québec (1960 à 1964) : les contraintes économiques et les enjeux culturels. Dans C. Baudoux (dir.), Féminisation et masculinisation de la gestion, Cabiers de recherche du GREMF, 29.

Fortin, R. (2006). L'insertion professionnelle des nowvelles directions d'établissement d'enseignement. Fédération québécoise des directions d'établissement d'enseignement. http:// fqde.qc.ca/wp-content/uploads/2013/09/Insertionjanvier2006.pdf

Gosselin, G. et Lessard, C. (2008). Les deux principales réformes de l'éducation du Québec moderne : témoignages de ceux et celles qui les ont initiées. PUL.

Gravelle, F. (2012). Quels sont les principaux changements qui ont modifié la fonction de direction ou de direction adjointe d'établissement scolaire depuis l'avènement de la réforme de l'administration publique au Québec? Éducation et francophonie, 40(1) 76-93. https://doi.org/10.7202/1010147ar

Guichard-Claudic, Y., Kergoat, D. et Vilbrod, A. (dir.). (2008). L'inversion du genre : quand les métiers masculins se conjuguent au féminin ... et réciproquement. Presses universitaires de Rennes.

Héon, L. et Kamyap, R.-V. (2018). La gestion des ressources humaines en éducation et le genre. Dans J. Moisset, P. Toussaint et J. Plante (dir.), La gestion des ressources humaines pour la réussite scolaire (p. 433-466). PUQ.

Héon, L., Lapointe, C. et Langlois, L. (2007). Réflexions sur le leadership des femmes et des hommes en éducation. Recherches féministes, 20(1) 83-99. https://doi.org/10.7202/016117ar

Héon, L., Lapointe, C. et Langlois, L. (2008). Le leadership des femmes et des hommes : ni rose, ni bleu, mais plutôt lilas. La féminisation de la gestion. Le Point en administration de l'éducation, 11(1), 26-29.

Héon, L. et Mayrand, N. (2003). L'accès des femmes aux postes de direction dans des écoles secondaires de Montréal. Recherches féministes, 16(1) 159-185. https://doi.org/10.7202/007346ar

Kelleher, F. (2011). Women and the teaching profession: Exploring the feminisation debate. Commonwealth Secretariat et UNESCO.

Lapeyre, N. (2005). Féminisation = dévalorisation? Chantiers politiques, 3, 93-104.

Lapeyre, N. (2006). Les professions face aux enjeux de la féminisation. Éditions OCTARÈS.

Malochet, G. (2005). Guillaume Malochet dans l'ombre des hommes : la féminisation du personnel de surveillance des prisons pour hommes. Societés contemporaines, 59-60(3-4), 199-220. https://doi.org/10.3917/soco.059.0199

Malochet, G. (2007). La féminisation des métiers et des professions : quand la sociologie du travail croise le genre. Sociologies pratiques, 14, 91-99. https://doi.org/10.3917/sopr.014.0091

Ministère de l'Éducation du Québec (MEQ). (1997). Prendre le virage du succès : plan d'action ministériel pour la réforme de l'éducation. Gouvernement du Québec. https://collections.banq.qc.ca/ark:/52327/bs40383 
Ministère de l'Éducation, du Loisir et du Sport (MELS). (2006). Étude des pratiques de soutien et d'accompagnement des nouvelles directions d'établissement [version abrégée]. Gouvernement du Québec. http://www.education.gouv.qc.ca/fileadmin/site_web/ documents/reseau/formation_titularisation/EtudePratiqueSoutienAccomp_abr_f.pdf

Ministère de l'Éducation, du Loisir et du Sport (MELS). (2008). La formation à la gestion d'un établissement d'enseignement : les orientations et les compétences professionnelles. Gouvernement du Québec. http://www.education.gouv.qc.ca/fileadmin/site_web/ documents/reseau/formation_titularisation/07-00881.pdf

Ministère de l’Éducation, du Loisir et du Sport (MELS). (2015). PERCOS-Bloc 2 2014-2015. Gouvernement du Québec.

Ouellette, R. (2000). Prévisions de l'effectif enseignant des commissions scolaires de 1996-1997 à 2010-2011. Direction des statistiques et études, ministère de l'Éducation du Loisir et du Sport.

Pelletier, G. (2001). La relève des dirigeants scolaires au Québec. La revue des échanges, 18(70), 26-27.

Poirel, E. et Yvon, F. (2009). Le stress des directions d'école an Québec. Association montréalaise des directions d'établissement scolaire.

QUÉBEC. Loi modifiant la Loi sur l'instruction publique et diverses dispositions législatives (RLRQ), [Québec], Éditeur officiel du Québec, 1997. file://C:/Users/STPHAN 1/AppData/Local/Temp/MicrosoftEdgeDownloads/ c04577ea-3285-4dc3-80df-ce439eff7d73/pl_97_180f.pdf

QUÉBEC. Règlement sur les conditions d'emploi des gestionnaires des commissions scolaires, 1998 GOQ 2, 5498 et 6061, RLRQ [Québec], Éditeur officiel du Québec, 2001. https://www.canlii.org/fr/qc/legis/regl/1998-goq-2-5498-et-6061/derniere/1998-goq-2-5498-et6061.html

St-Germain, M. (2011). Le leadership et la gestion du temps. Dans J. Rocque (dir.), La direction d'école et le leadership pédagogique en milieu minoritaire (p. 219-256.). Presses universitaires de Saint-Boniface.

St-Germain, M. (2013). Étude des divergences entre le temps de travail réel et le temps de travail soubaité chez les directions membres de la FQDE [version abrégée]. Fédération québécoise des directions d'établissement d'enseignement. http://www.fcdef.ca/wp-content/uploads/2015/11/Michel-St-Germain-version-abregee.pdf

Zaidman, C. (1986). La notion de féminisation : de la description statistique à l'analyse des comportements. Dans N. Aubert et V. Gaulejac (dir.), Le sexe du pouvoir : femmes, hommes et pouvoirs dans les organisations (p. 281-288). Desclée de Brouwer. 